\title{
Percolation of spatially constrained Erdős-Rényi networks with degree correlations
}

\author{
C. Schmeltzer, ${ }^{1}$ J. Soriano, ${ }^{2}$ I. M. Sokolov, ${ }^{1}$ and S. Rüdiger ${ }^{1, *}$ \\ ${ }^{1}$ Institut für Physik, Humboldt-Universität zu Berlin, 12489 Berlin, Germany \\ ${ }^{2}$ Departament d'ECM, Facultat de Física, Universitat de Barcelona, 08028 Barcelona, Spain
}

(Received 12 August 2013; published 13 January 2014)

\begin{abstract}
Motivated by experiments on activity in neuronal cultures [J. Soriano, M. Rodríguez Martínez, T. Tlusty, and E. Moses, Proc. Natl. Acad. Sci. 105, 13758 (2008)], we investigate the percolation transition and critical exponents of spatially embedded Erdős-Rényi networks with degree correlations. In our model networks, nodes are randomly distributed in a two-dimensional spatial domain, and the connection probability depends on Euclidian link length by a power law as well as on the degrees of linked nodes. Generally, spatial constraints lead to higher percolation thresholds in the sense that more links are needed to achieve global connectivity. However, degree correlations favor or do not favor percolation depending on the connectivity rules. We employ two construction methods to introduce degree correlations. In the first one, nodes stay homogeneously distributed and are connected via a distance- and degree-dependent probability. We observe that assortativity in the resulting network leads to a decrease of the percolation threshold. In the second construction methods, nodes are first spatially segregated depending on their degree and afterwards connected with a distance-dependent probability. In this segregated model, we find a threshold increase that accompanies the rising assortativity. Additionally, when the network is constructed in a disassortative way, we observe that this property has little effect on the percolation transition.
\end{abstract}

DOI: 10.1103/PhysRevE.89.012116

PACS number(s): 64.60.ah, 87.85.dq, 05.70.Fh, 64.60.an

\section{INTRODUCTION}

The theory of percolation, originally introduced for the mathematical description of fluid flow through random porous materials [1], possesses great potential to investigate systems with random connectivity [2]. Percolation theory has been applied to many natural and technological systems, such as electrical conductivity [3], cellular chemical patterns [4], immune networks [5,6], epidemics [7], or social networks [8,9]. A pivotal question regards the connectivity or the number of links in a network at which a giant component emerges since many structural and dynamical properties of the network undergo a rapid transition at this point $[10,11]$. More importantly, the features of the transition may convey information on the major topological traits of the underlying network. These aspects have conferred percolation approaches a remarkable predicting power, particularly in living systems.

Recently, the activity in networks of cultured neurons [12] has been studied in terms of this theory, with the neuronal synapses identified as links between cells, and the unison firing of neurons in the culture identified as percolation [13-16]. The scaling of the giant component has been used to quantify the development of connections [14], topological properties of the underpinned circuitry $[13,15,17]$, as well as their interplay with the observed neuronal dynamics $[14,18]$.

The first random network of which the percolation threshold has been calculated exactly in the limit of infinitely large size is the Erdôs-Rényi graph (ER) [19]. The possibility of an analytical treatment using a mean-field description makes this simple model particularly attractive. However, several systems, and most notably neuronal tissues, are embedded in a physical space. The connection between spatially close nodes is often more probable than between distant nodes $[7,14,20]$,

\footnotetext{
*sten.ruediger@physik.hu-berlin.de
}

therefore directly affecting the degree distribution, average path length, and clustering coefficient of random networks [21-24] (see also [25,26] for reviews). In neuronal systems, it has been shown that spatial constraints delineate several topological properties of functional brain networks [27]. Furthermore, metric correlations, inherited from the spatial embedding of neurons and connections, play an important role in shaping the collective spontaneous dynamics in neuronal cultures [28].

Modeling spatial networks is challenging. In many studies, the connection probability between two nodes $i, j$ of a random network is given by a function of the link length between them $f\left(l_{i j}\right)$, which is often chosen to be a power law $f\left(l_{i j}\right)=l_{i j}^{\alpha}$. Power-law length distributions of connections have been empirically observed in social networks [29-31], the Internet [32], and air transportation networks [33,34].

In addition to the metric constraints, degree correlations have also been shown to affect the percolation behavior of random networks [35-42]. A prominent example of such correlations is assortativity, which describes networks in which nodes preferably attach to other nodes with similar degree $[35,36]$. Detailed criteria for the relevance of degreecorrelations to the percolation threshold were calculated by the authors of [42]. Assortativity is believed to play an important role in brain networks since it may provide a subnetwork of highly connected nodes where information flow can be reorganized in case of insult [43]. Assortativity in ER networks was shown to change the universality class of the percolation transition, while disassortativity was found to be irrelevant in this regard [41].

In this work we investigate the impact of degree correlations on the percolation behavior of a spatial random network with Poisson degree distribution. We find that assortativity is related to strong changes in the percolation behavior. We employ two different methods to achieve degree correlations. In the first method, nodes are homogeneously distributed 
in a spatial domain and degree correlations are enforced by the wiring probability during network construction. This probability depends both on distance and the degrees of nodes to be linked. In the second method, nodes are first spatially shuffled in dependence on their degree. This results in networks with homogenous density of nodes, but segregation in terms of the node degrees. The nodes are finally linked in a distance-dependent manner. In this way, degree correlations occur only for spatially constrained networks.

Our study results in two major conclusions. First, in our nonsegregated spatial networks, assortativity leads to a decrease of the percolation threshold, as expected from prior results for nonspatial networks [44]. Second, in segregated networks, we find a threshold increase that accompanies the rising assortativity. Interestingly, the segregated case could be relevant for neuronal networks, where the density of neurons may be approximately constant in space, but the number of links per neuron may be space dependent, as possibly occurs in modular brain networks [27]. If we interpret percolation as a conveyance of information across a neuronal network, our results suggest that the transmission is eased by a simple assortative network, but hindered by a network with degree segregation.

\section{SPATIAL EMBEDDING OF THE RANDOM NETWORK}

We first describe the construction of spatial networks without degree correlations. To construct an undirected network with the desired degree distribution, we use the configuration model of Newman, Strogatz, and Watts [45]: Each node is assigned a target-degree, drawn from a desired degree sequence. The target-degree of a node can be regarded as a number of stubs, which will be randomly connected to stubs of other nodes. We focus on the Poisson degree distribution of the ER graph [46]. For convenience, we quantify network connectivity with the ratio of the total number of links and the number of nodes $q=E / N$, which is related to the mean degree of the network $\langle k\rangle=2 q$. In our computational network construction, the nodes are positioned randomly in a domain of side lengths $L \times L$ with $L=100$. Then, we randomly choose a free stub (belonging to node $i$ ) and calculate the probabilities $\Pi$ to connect to remaining free stubs in the network

$$
\Pi(\text { free stub at node } j)=\frac{l_{i j}^{\alpha}}{Z},
$$

where $l_{i j}$ is the Euclidian distance between the nodes and $Z=\sum l_{i j}^{\alpha}$ a normalization constant, summing over all remaining stubs that node $i$ can connect to. Self-loops and multiedges are rejected in the algorithm. A stub from node $j$ is randomly chosen according to this distribution and the procedure is iterated until all stubs are connected.

The exponent $\alpha$ plays the role of a distance exponent. For $\alpha=0$ there are no metric correlations in the network. Long-range connections gradually become less probable as $\alpha$ decreases below zero, until the network is essentially described by neighbor-to-neighbor interactions for $\alpha \ll-1$.

Examples of networks constructed in this way for different values of the exponent $\alpha$ are shown in Fig. 1. The networks

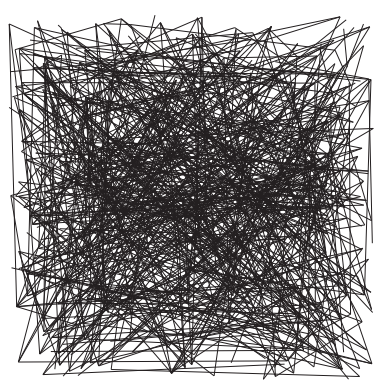

(a)

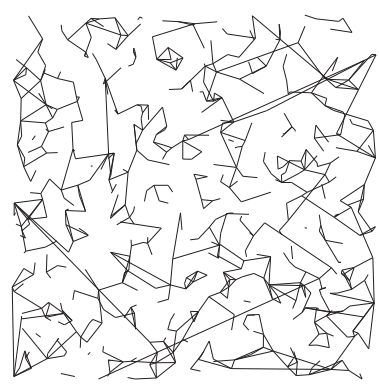

(b)
FIG. 1. Illustrative network with 300 nodes and 600 links. (a) Random network without spatial dependence $\alpha=0$. (b) Spatially constrained network with $\alpha=-10$. Note that for $\alpha=-10$ connectivity is mostly local. Nodes are not shown for clarity.

illustrate that for large $|\alpha|$ the desired distribution of link lengths cannot be achieved since the probability of long-range links is very small. The construction algorithm provided by Eq. (1) gives rise to a distribution of links lengths $P(l)$, as shown in Fig. 2. As we will elaborate below, only for small $|\alpha|$ the probability distribution of link lengths follows a power-law scaling function, of the form

$$
P(l) \sim l^{\alpha+1} .
$$

The exponent $\alpha+1$ originates from the annulus area $2 \pi l d l$ in the relation $P(l) d l \sim l^{\alpha}(2 \pi l d l)$. Due to the fixed degree of each node, the length distribution in our simulations cannot follow arbitrarily large negative exponents: As $\alpha$ decreases, nodes will preferably connect to others in their close neighborhood. As the wiring procedure progresses, many nodes reach their target degree and will be rejected from

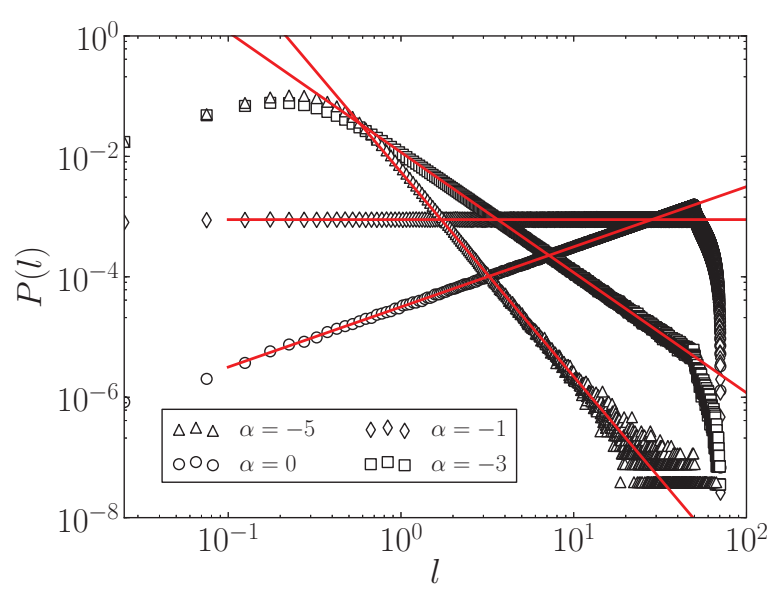

FIG. 2. (Color online) Distribution of link lengths in spatial networks generated with different distance exponents $\alpha$. Gray (red) straight lines are fits of the form $P(l) \sim l^{\delta}$, with $\delta=1.0,0.0,-2.0$, and -3.4 , from the largest to the smallest slope, respectively. Note that $\alpha$ is the target exponent of the network, while $\delta$ is the resulting exponent of the actual network. $\delta=\alpha+1$ for $\alpha>-4$, but for smaller values of $\alpha$ it holds that $\delta>\alpha+1$. In all cases the distributions are constructed with $10^{5}$ nodes and network connectivity $q=2$ and after averaging over 100 realizations. 
connection. Thus, nodes in the close neighborhood might not be available and links will extend to more distant areas.

To quantify the limitation in obtaining the desired distribution, we denote by $\delta$ the exponent from the distribution $P(l) \sim l^{\delta}$ obtained by fitting the tail of the length distribution in our simulations. These fits are shown as gray (red) straight lines in Fig. 2. We observed that the equality $\delta=\alpha+1$ holds only for small values of $\alpha$, up to $\alpha \simeq-3$, and fails for larger negative values. We note that for exponent $\alpha \ll-3$, the nodes will essentially connect to their nearest neighbors, giving rise to practically the same distributions independently on $\alpha$. This "saturated distribution" is indeed characterized by an exponent of -3 , a value that can be estimated as follows.

We consider an extreme scenario, where a random stub at iteration step $m$ is being connected to the nearest node that has a free stub. The probability distribution of the distance $l$ of the connection is then

$$
P_{m}(l) d l=Z(m)\left[1-P_{\text {full }}(m)\right]\left[P_{\text {full }}(m)\right]^{n(l)} l d l,
$$

where $P_{\text {full }}$ is the probability of a node to have reached its target degree and $n(l)=c \pi l^{2}$ is the mean number of nodes in the circle of radius $l$. Here, $c$ denotes the node density (total number of nodes divided by the domain area). The prefactor $Z(m)$ ensures normalization

$$
\int_{0}^{\infty} P_{m}(l) d l=1 .
$$

At each iteration step $m$ two remaining stubs in the network will be connected, until all nodes arrive at their target-degree. The probability of a random node to have reached its targetdegree will be a monotonically increasing function from 0 to 1 with increasing $m$. As a simple estimate, we choose $P_{\text {full }}=m / M$, with $M$ being the target number of links. By inserting this assumption in Eqs. (3) and (4), we find

$$
\begin{gathered}
P_{m}(l) d l=Z(m)(1-m / M)(m / M)^{c \pi l^{2}} l d l, \\
Z(m)=\frac{2 \pi c M}{m-M} \ln (m / M) .
\end{gathered}
$$

The total probability density of a link to be of length $l$ is then

$$
P(l)=\frac{1}{M} \sum_{m=0}^{M} P_{m}(l) .
$$

For large $M$, we can approximate the sum by the integral

$$
P(l)=\frac{1}{M} \int_{0}^{M} P_{m}(l) d m .
$$

Solving the integral with Eqs. (5) and (6) inserted, we obtain

$$
P(l)=\frac{4 \pi l c}{\left(1+\pi c l^{2}\right)^{2}} .
$$

In the limit of small and large $l$, this finally yields

$$
P(l) \sim \begin{cases}l, & l \ll 1, \\ l^{-3}, & l \gg 1 .\end{cases}
$$

This analysis confirms the saturation value of $\delta=-3$ for large $l$. Figure 3 illustrates the dependence of $\delta$ on $\alpha$ and the saturation at $\delta \simeq-3$ for $\alpha \lesssim-4$.

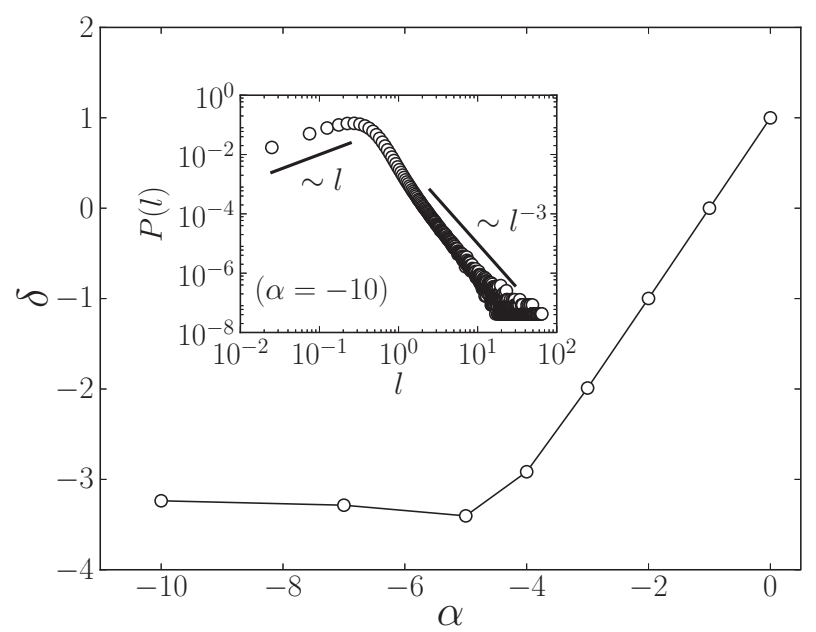

FIG. 3. Relation between the exponents of the preset link length distribution $P(l) \sim l^{\alpha+1}$ and the actual distribution $P(l) \sim l^{\delta}$ after network construction. The main plot shows that the relation $\delta=\alpha+1$ of Eq. (2) remains valid down to $\alpha \approx-4$. For values below -4 , the exponent $\delta$ saturates to $\approx-3$. The inset shows the probability distribution of link lengths for $\alpha=-10$. Lines are a guide to the eye to show the scaling of the distribution at the limit of small and large $l$, see Eq. (10).

\section{MODELING DEGREE CORRELATIONS}

We use two different methods to generate degree-correlated embedded networks. In the first method, degree correlations are enforced directly by a degree-dependent prefactor of the connection probability. In the second method, nodes are first spatially segregated by their degree and then connected in the same way as in the uncorrelated network.

\section{A. Model 1: Degree-dependent connection probability}

In this model, nodes are first randomly distributed in the spatial domain. Then, two random nodes $i$ and $j$, separated by a distance $l_{i j}$, are connected with probability

$$
\Pi\left(l, k_{i}, k_{j}\right) \sim e^{\rho\left|k_{i}-k_{j}\right|} l_{i j}^{\alpha},
$$

where $k_{i}, k_{j}$ are their target-degrees, $\alpha$ is the connectivity exponent introduced before, and $\rho$ is a prefactor which determines the type and strength of the degree correlations. When the exponent is negative, nodes preferably connect to nodes with similar target-degree which results in assortativity, whereas a positive exponent leads to disassortativity. We measure degree correlations with the Pearson coefficient $r$ as defined in [35]

$$
r=\frac{1}{\sigma^{2}} \sum_{j k} j k\left(e_{j k}-Q_{j} Q_{k}\right),
$$

where $e_{j k}$ is the joint probability distribution of the remaining degrees $j$ and $k$ of two vertices at either end of a randomly chosen edge and $Q_{k}=\sum_{j} e_{j k}=\sum_{j} e_{k j}$. The Pearson coefficient is normalized by the variance $\sigma^{2}=\sum_{k} k^{2} Q_{k}-\left(\sum_{k} k Q_{k}\right)^{2}$ and ranges from -1 for a fully disassortative network to 1 for a fully assortative network. In Fig. 4, the value of $r$ is shown for networks with different combinations of 


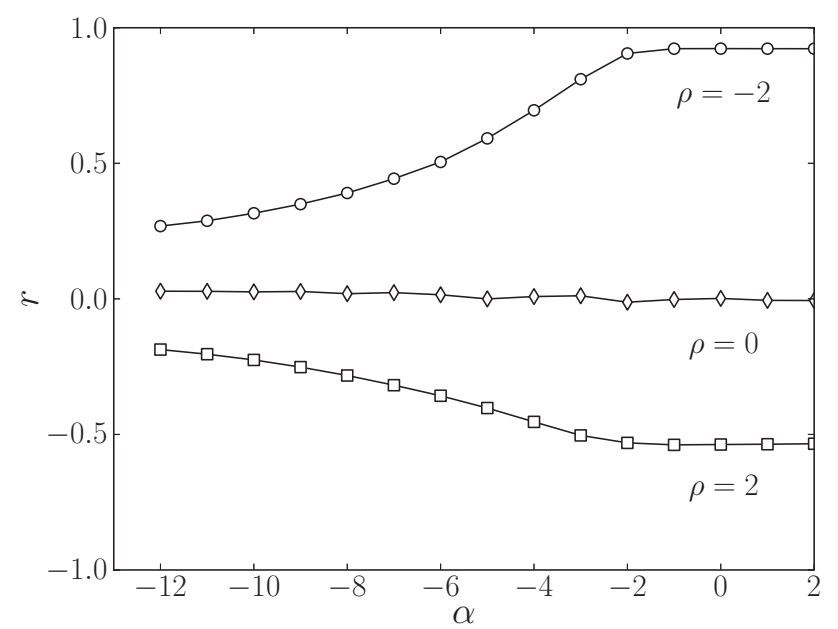

FIG. 4. Pearson degree correlation coefficient $r$ for spatial networks with $10^{5}$ nodes and different exponents $(\alpha, \rho)$. $(\bigcirc)$ Assortative network, $(\diamond)$ uncorrelated network, $(\square)$ disassortative network.

the exponents $(\alpha, \rho)$. Note that spatial constraints also limit the possibility of imposing degree correlations, indicated by a decreasing Pearson coefficient for decreasing $\alpha$. In the following discussions of model 1 , we choose $\rho= \pm 2$ for assortative and disassortative networks, respectively, if not stated otherwise.

\section{B. Model 2: Spatial segregation of node degrees}

In the second model, the nodes are spatially segregated according to their target-degree before they are connected. The spatial segregation is achieved by a swapping algorithm similar to the model of Badham et al. [47]: First, the mean targetdegrees $\bar{k}_{i}, \bar{k}_{j}$ within distance $d$ of two randomly selected nodes is calculated. The radius $d$ is defined so that a circle of this radius contains on average as many nodes as the larger target-degree of the two nodes: $\pi d^{2} c=\max \left(k_{i}, k_{j}\right)$, with node density $c=N / L^{2} . N$ and $L^{2}$ are, respectively, the number of nodes in the network and its area. The target-degree of both nodes is swapped if

$$
\left(k_{i}-\bar{k}_{i}\right)^{2}+\left(k_{j}-\bar{k}_{j}\right)^{2}>\left(k_{i}-\bar{k}_{j}\right)^{2}+\left(k_{j}-\bar{k}_{j}\right)^{2},
$$

thus, sorting nodes of similar target-degrees together. The swapping procedure is illustrated in Fig. 5. The nodes are then connected in the same way as in the uncorrelated spatial network with connection probability $\Pi(l) \sim l_{i j}^{\alpha}$. Note that the swapping procedure changes the topology of the network while maintaining the node density, as illustrated in Fig. 6. We found that a number of iterations $S \approx 10^{3} \mathrm{~N}$ are needed until the network reaches a stationary state, e.g., the Pearson coefficient saturates (Fig. 7). Assortativity emerges for $\alpha<0$ because nodes preferably connect to nodes with a similar degree in their neighborhood, as shown in Fig. 8. While this model worked well for generating assortative networks, we found that it was not able to produce disassortative ones. Therefore, disassortative networks will only be discussed in the framework of model 1.

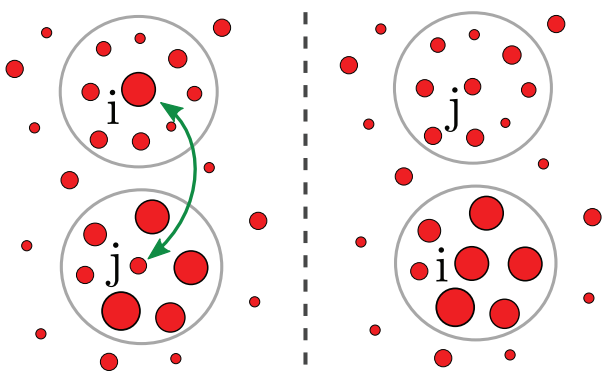

FIG. 5. (Color online) Schematic representation of the swapping procedure for two nodes $i$ and $j$. The large gray circle shows the swapping neighborhood range of radius $d$. Gray (red) dots depict nodes, with the size proportional to their target-degree. The positions of the nodes $i$ and $j$ (left) are swapped only if the swapping operation increases the difference between the mean target-degree of the two neighborhoods (right).

\section{FINITE-SIZE SCALING METHOD}

To quantitatively study the percolation behavior of the described networks, we employ standard critical scaling theory. It is well known that the behavior close to the percolation transition is associated with power-law scaling [48]. Close to the percolation threshold $q_{c}$, the size of the giant component $G$ and the average size of finite clusters $S$ scale as

$$
\begin{gathered}
G \sim\left(q-q_{c}\right)^{\beta}, \quad q>q_{c}, \\
S \sim\left|q-q_{c}\right|^{-\gamma} .
\end{gathered}
$$

To obtain the characteristic percolation exponents and thresholds, we use the finite-size scaling method. In infinite networks,

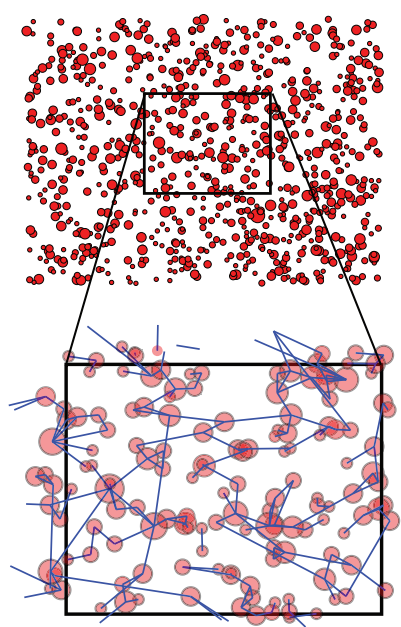

(a)

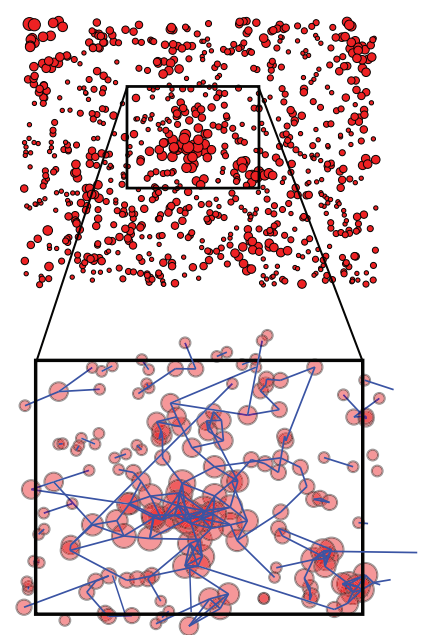

(b)
FIG. 6. (Color online) Comparison of the topological structure of models 1 and 2, for networks with $N=10^{3}$ nodes (red spots) and $\alpha=-10$. Spot size is proportional to the degree of the node. Blue lines are representative links between nodes. (a) Construction using a homogeneous distribution with no swapping (model 1). (b) Segregated construction (model 2 ) after $10^{6}$ node swaps, illustrating the emergence of highly connected node assemblies. 


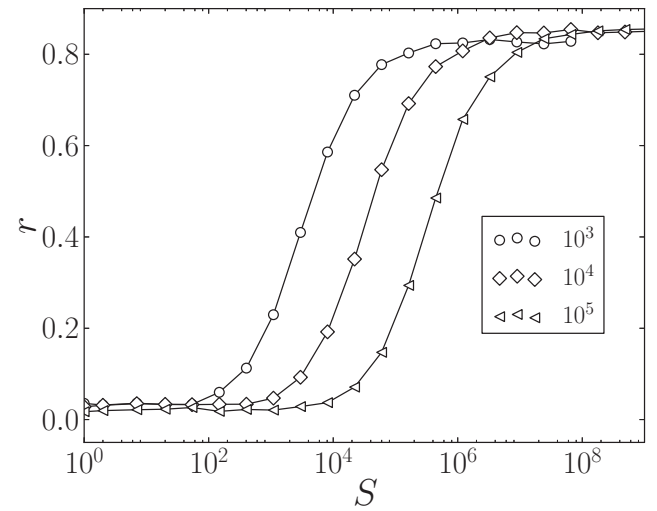

FIG. 7. Pearson degree correlation coefficient for networks of different size with $\alpha=-5$ after $S$ iterations of the swapping algorithm. About $10^{3} \mathrm{~N}$ iterations are needed for the Pearson coefficient to saturate.

the average size of finite clusters diverges at the percolation threshold, whereas in finite networks it scales with [48,49]

$$
S(q, N)=N^{\frac{\gamma}{v d}} F\left(\left(q-q_{c}\right) N^{\frac{1}{v d}}\right),
$$

where $d$ is the spatial dimension of the system. For spatially embedded random networks, it has been shown that a useful definition of $d$, different from the embedding space dimension, can be given [50], and a connection to classical percolation scaling theory can be drawn based on this definition. A different case is that of the nonspatial complex networks, where it has been proposed that the product $v d$ can be replaced by $\bar{v}$ in the case that the criticality belongs to the mean-field universality class [51]. In our scaling analysis below we will therefore consider only the combined expressions $\gamma / \nu d$ and $1 / v d$. The scaling function $F(x)$ has the limiting behavior $F(|x| \rightarrow 0) \rightarrow c_{1}$ and $F(|x| \gg 1) \rightarrow x^{-\gamma}$ with a constant $c_{1}$. Thus, close to the percolation threshold, we obtain for the

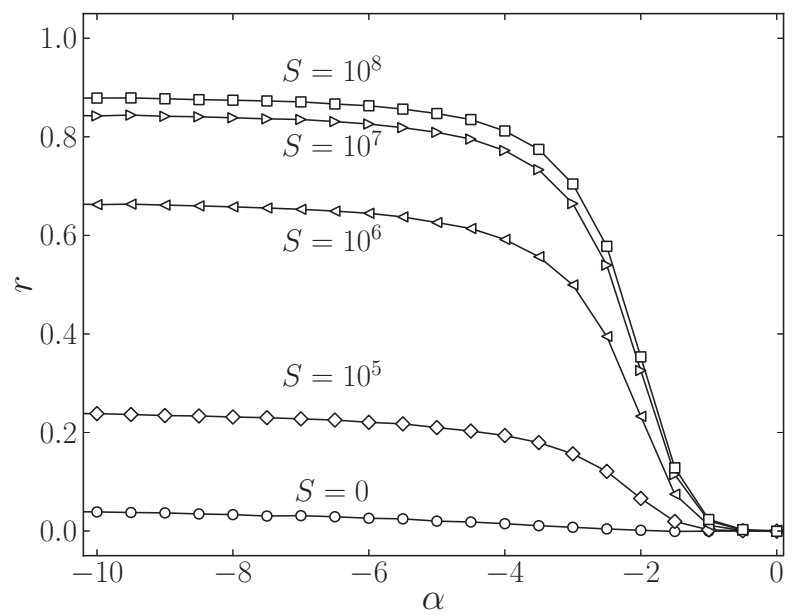

FIG. 8. Pearson degree correlation coefficient $r$ for model 2 networks with spatial segregation and $N=10^{5}$, for different number of node-swaps $S$. Assortativity emerges for large negative distance exponents $\alpha$.
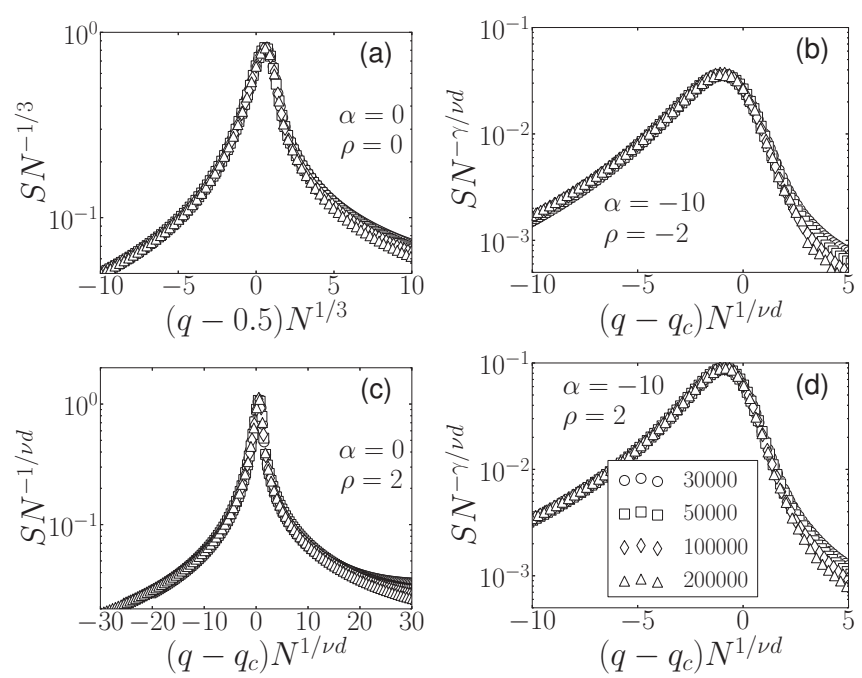

FIG. 9. Finite-size scaling of mean cluster size of model 1 networks for different values of $\alpha$ and $\rho$. Data points collapse into a single curve. The set of percolation threshold and exponents $q_{c}, \frac{1}{v d}$, and $\frac{\gamma}{v d}$ were then obtained by the fitting procedure described in the main text. For each plot, their values are (a) $q_{c}=0.5, \frac{1}{v d}=0.33$, $\frac{\gamma}{v d}=0.33$; (b) $q_{c}=0.82, \frac{1}{v d}=0.35, \frac{\gamma}{v d}=0.87$; (c) $q_{c}=0.55$, $\frac{1}{v d}=0.33, \frac{\gamma}{v d}=0.33$; and (d) $q_{c}=0.96, \frac{1}{v d}=0.35, \frac{\gamma}{v d}=0.85$.

average size of finite clusters

$$
S(q, N) N^{-\frac{\gamma}{v d}} \sim N^{\frac{1}{v d}}\left(q-q_{c}\right) .
$$

At $q=q_{c}$, the giant component mass $N G(N)$ scales with $[48,49]$

$$
N G(N) \sim N^{\frac{D}{d}}
$$

where $D$ is the fractal dimension of the system that is related to the scaling exponents by the hyperscaling relation $[48,49]$

$$
\frac{D}{d}=\frac{1}{2}\left(1+\frac{\gamma}{\nu d}\right) .
$$

The original ER graph belongs to the same universality class as the mean-field transition [19]. In the limit of large network sizes, the percolation exponents and thresholds can be calculated analytically. The exponents of this universality class are $\gamma=1$ and $d v=3$, while the value of the percolation threshold depends on the scaling of the connectivity. In our case we have $q_{c}=0.5$.

Using the relation of Eq. (17) for the average size of finite clusters $S(q, N)$, we obtain the percolation threshold $q_{c}$ as well as the exponents $\gamma$ and $\nu d$. This is achieved by plotting the rescaled $S(q, N)$ close to the percolation threshold $q_{c}$ for different network sizes $N$. The percolation threshold and exponents are then fitted, providing a collapse of the data points into a single curve. In Fig. 9 we show exemplary plots of the rescaled mean cluster size using the relation of Eq. (17). Data points collapse into a single curve, indicating that the scaling exponents and percolation threshold are reliable.

From Eq. (19), the exponents $\gamma$ and $v d$ can then be used to calculate the fractal dimension $D / d$, which will be used to characterize the percolation transition. 

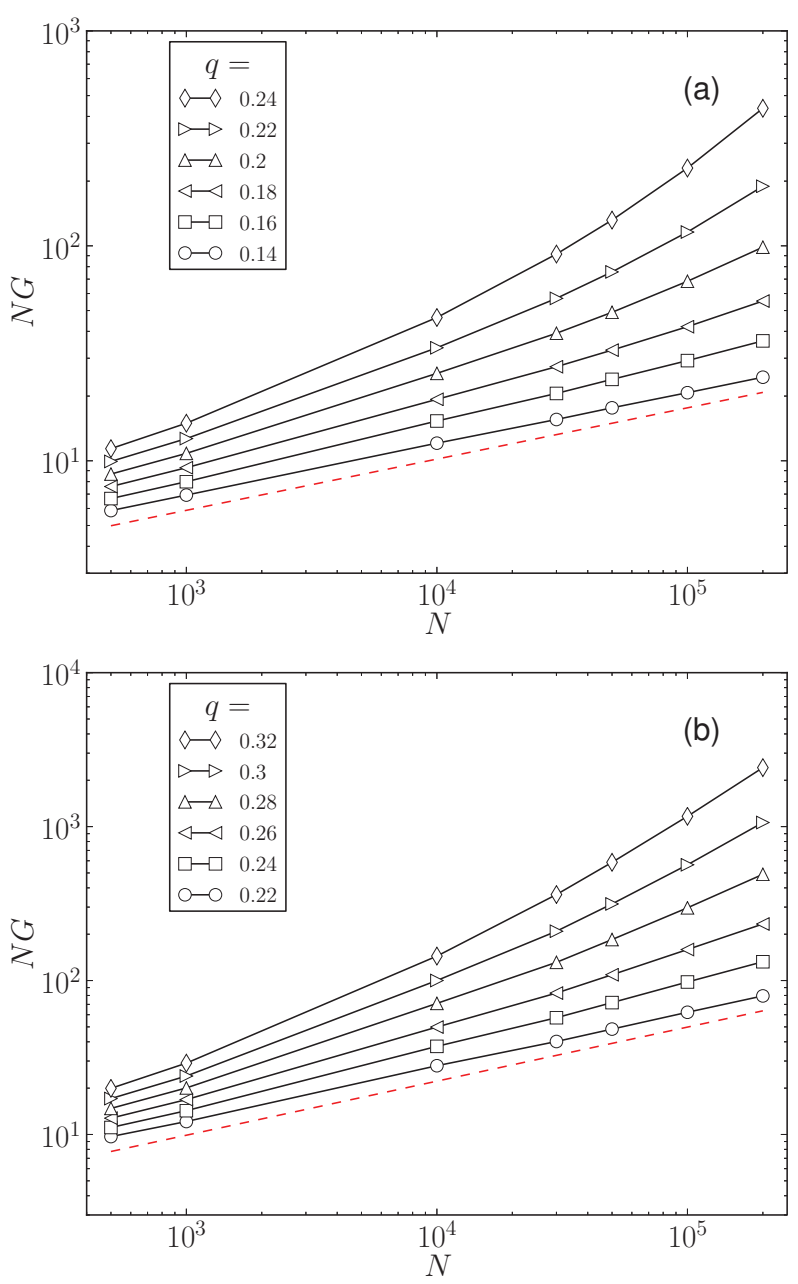

FIG. 10. (Color online) Scaling of giant component mass $N G$ at different $q$ for an assortative model 1 network with $\rho=-2$ for (a) $\alpha=0$ and (b) $\alpha=-3$. The gray (red) dashed lines are guides to the eye.

It was shown by Noh [41] that in highly assortative ER networks the relation of Eq. (17) for the mean cluster size is not valid. However, these networks still show power-law scaling for the giant component. Interestingly, this scaling exists not only at $q_{c}$, but below the threshold as well

$$
N G(q, N) \sim N^{\kappa(q)} \text { for } q \leqslant q_{c} .
$$

The property that the scaling does not appear above $q_{c}$ will be used to estimate the critical transition point $q_{c}$, as follows. We plotted $N G(q, N)$ as a function of $N$ for successively smaller $q$ values, and chose $q_{c}$ to be the value where the power-law dependence emerges, which is indicated by a linear behavior in a log-log plot (Fig. 10). A linear log-log graph emerges for the lowest $q$ value in the plots, indicating the correct power-law dependency of Eq. (20). This method is indeed a valuable tool to characterize the percolation threshold of spatial networks, as shown by Li et al. [44]. The values of $q_{c}$ estimated by this method are consistent with those obtained by the finite-size scaling (data not shown). However, we found the finite-size scaling method to be much more precise.

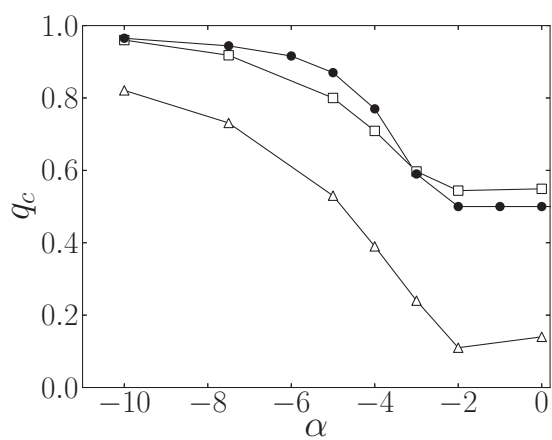

FIG. 11. Percolation threshold for different values of $\alpha$ for uncorrelated networks $(\bullet, \rho=0)$, compared to correlated networks generated with model 1: assortative networks $(\triangle, \rho=-2)$ and disassortative networks $(\square, \rho=2)$. Uncorrelated and disassortative networks show a similar trend, while assortative networks display a significant decrease of the percolation threshold compared to the others.

\section{RESULTS}

\section{A. Uncorrelated spatial networks}

We first consider the uncorrelated embedded network. The solid circles in Figs. 11 and 12 show the dependence of $q_{c}$ and $D / d$ on the distance exponent $\alpha$. For $\alpha$ larger than -2 , we find numerically $\frac{D}{d}=0.66$ and $q_{c}=0.5$. Using Eq. (19) these values correspond to the expected results for the universality class of random ER graphs with mean-field behavior $(\gamma=1, v d=3$, [51]). For $\alpha<-2$ in Fig. 11, we observe a positive shift of the percolation threshold. For strong constraints $(\alpha<-5)$, we calculated $\frac{D}{d}=0.945$, which is close to the fractal dimension for regular two-dimensional lattices $D / d \simeq 0.95$. In the intermediate region $-5<\alpha<-2$ the values of the fractal dimension are in between (Fig. 12). This result indicates a shift of the percolation transition from the universality class of random networks to the universality class of two-dimensional lattices.

Similar results were found for spatial ER networks on a grid by Li et al. [44]. Note that the fractal dimension shown

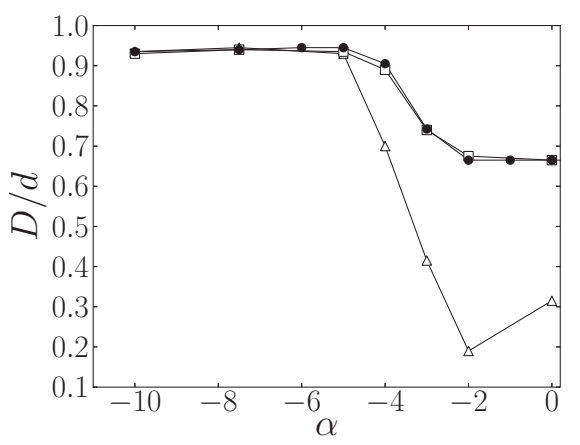

FIG. 12. Fractal dimension at criticality for different values of $\alpha$ for uncorrelated networks $(\bullet)$, compared to correlated networks generated with model 1: assortative networks $(\triangle)$ and disassortative networks $(\square)$. Only assortative networks show a significant decrease in the fractal dimension, which saturate for all network constructions at $\alpha \leqslant-5$. 
in Fig. 12 saturates for values of $\alpha<-5$, which is the value where the exponent of the link length distribution saturates (Fig. 3), whereas the percolation threshold still changes for values $\alpha<-5$.

\section{B. Model 1: Correlated spatial networks with degree-dependent connection probability}

We now investigate the impact of degree correlations on the percolation transition in embedded networks (open symbols in Figs. 11 and 12). The corresponding Pearson coefficients for networks with $\rho=-2$ and 2 were previously described in Fig. 4(a). Exemplary plots of the dependence of the giant component and the mean cluster size on connectivity, for $N=10^{5}$ and different values of $\alpha$, are shown in Fig. 13. For $\alpha=0$, assortativity decreases the percolation threshold, while disassortativity slightly increases it.

An evaluation using the finite-size scaling method reveals that, similar to the uncorrelated case, spatial embedding $(\alpha<0)$ generally increases the percolation threshold in both correlated networks (assortative or disassortative, open symbols in Fig. 11). With assortativity, the percolation threshold always remains significantly below the uncorrelated case, indicating a persistent effect of positive degree correlations on the transition. However, disassortativity results in small changes of $q_{c}$. Clearly, the percolation threshold of the networks is affected by an interplay of both spatial constraints and degree correlations.

For both types of correlations we have also calculated the fractal dimension $D / d$ (open symbols in Fig. 12). Again, disassortativity has little effect, while for assortative networks and long-range connectivity $(0>\alpha>-5)$ we observe a
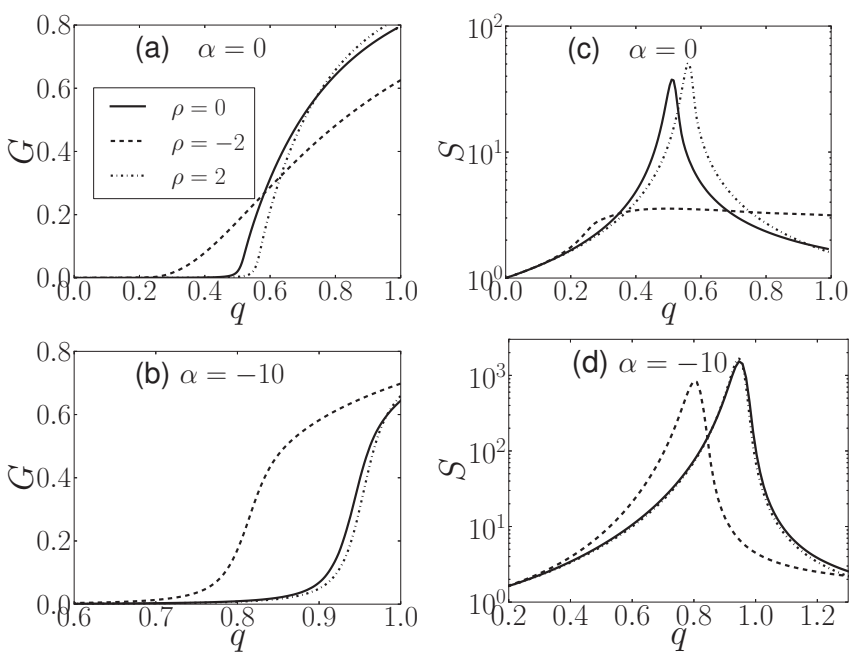

FIG. 13. Influence of $\alpha$ in the percolation threshold and mean cluster size for model 1 networks with $10^{5}$ nodes. (a,b) Size of the giant component for two extreme values of $\alpha$, corresponding to nonspatial networks $(\alpha=0)$ and spatial ones $(\alpha=-10)$, respectively. Three network configurations are compared: uncorrelated $(\rho=0)$, assortative $(\rho=-2)$ and disassortative $(\rho=2)$. Assortative networks always decrease the percolation threshold, i.e., less links are required to observe the emergence of a giant component. (c,d) Dependence of the mean cluster size on $\alpha$ for the same three networks.

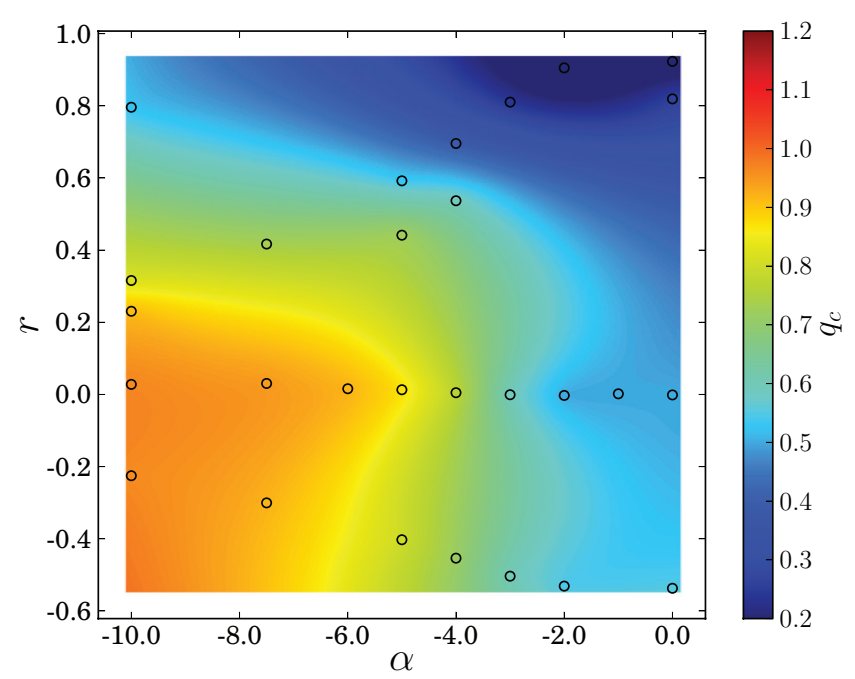

FIG. 14. (Color online) Dependence of the percolation threshold $q_{c}$ for model 1 networks on the Pearson coefficient $r$ and the distance exponent $\alpha$. Open symbols show the actual data points for the construction of the plot.

strong decrease of $D / d$ compared to the uncorrelated case. Similarly to the uncorrelated network, a transition regime exists for $-5<\alpha<-2$, where intermediate values of the fractal dimensions $D / d$ were found. For $\alpha<-5$, the percolation transition for both networks conforms to the universality class of two-dimensional lattices with $D / d=0.945$. It is interesting to note that, although the fractal dimensions of both networks are similar for $\alpha<-5$, the assortative network exhibits a much smaller $q_{c}$.

Figure 14 shows a contour plot of the dependence of the percolation threshold $q_{c}$ on the Pearson coefficient $r$ and the distance exponent $\alpha$. Clearly, the threshold-increasing effect of spatial constraints (large negative $\alpha$ ) competes with the threshold-decreasing effect of assortativity (large $r$ ). The plot is very illustrative to understanding the behavior of the constructed networks. Assortative networks with no spatial constraints $(\alpha=0)$ need in general less links to achieve global connectivity or, in other words, the giant component is more robust to removal of links. The addition of spatial constrains $(\alpha \lesssim 0)$ does not significantly modify the properties of the network as far as a the balance between short- and long-range connections is maintained (top-right corner of Fig. 14). Only for strong locality $(\alpha \ll-4)$ is the network compromised by a substantial increase in $q_{c}$. Disassortative networks, on the other hand, are always characterized by a high $q_{c}$ that increases even more when spatial constraints are introduced, revealing a high vulnerability of the giant component to link removal.

\section{Model 2: Assortative spatial networks with segregated degrees}

The network construction algorithm used in model 2 gives rise to topologies that are always assortative when locality is dominant $(\alpha \lesssim-2)$, as shown in Fig. 8. We note that for $\alpha=0$, 

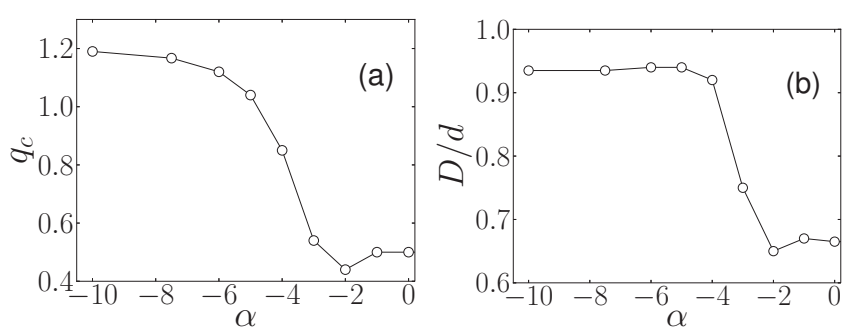

FIG. 15. Topological features of model 2 networks $\left(S=10^{8}\right)$, characterized by a spatial segregation of node degrees. (a) Percolation threshold as function of the distance exponent $\alpha$. (b) Fractal dimension at criticality as a function of $\alpha$.

the links are effectively random and the spatial segregation of node degrees does not affect network topology. Therefore, for $\alpha=0$, the network is uncorrelated and characterized by a mean-field percolation behavior.

As $\alpha$ decreases, both the percolation threshold and fractal dimension significantly increase. As shown in Fig. 15, the steep increase is followed by the saturation of the two numbers for $\alpha \lesssim-5$. The percolation threshold saturates at about $q_{c} \simeq 1.2$, a value that is significantly larger than the 0.95 observed in the uncorrelated case at saturation.

The contour plot of Fig. 16 shows that the thresholdincreasing effect of locality $(\alpha \ll-1)$ is intensified by assortative degree correlations (large $r$ ). Thus, networks with short-range links that are increasingly vulnerable to link removal become even more fragile. This behavior is contrary to model 1 networks, where assortativity enhances the robustness of the network. Note that in model 2 networks, assortativity can only emerge for nonrandom networks, e.g., for networks with high locality. Therefore, the top-right corner of Fig. 16 cannot be accessed by this model.

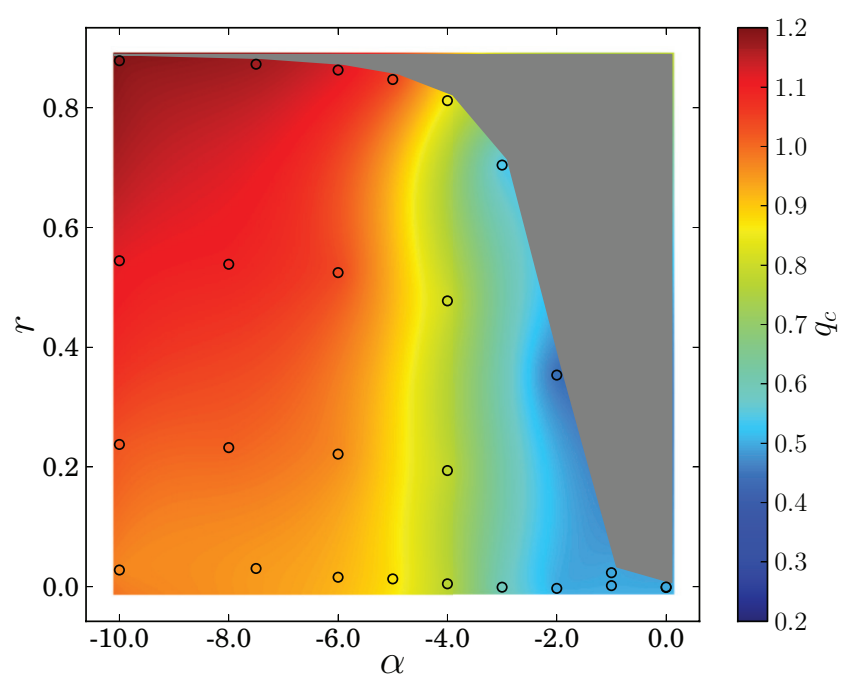

FIG. 16. (Color online) Dependence of the percolation threshold $q_{c}$ for model 2 networks on the Pearson coefficient $r$ and the distance exponent $\alpha$. Open symbols show the actual data points for the construction of the plot. Extrapolation to values that cannot be analyzed directly is omitted (gray area).

\section{Comparison of network models}

All of the three network architectures have in common a metric construction inherited from spatial constraints (incorporated through the "distance exponent" $\alpha$ ), but differ in the way additional node degree correlations are introduced. A first architecture is the simplest, uncorrelated one, with only spatial constraints. The other two comprise an assortative topology derived from degree correlations (model 1), and a topology that includes both node segregation and degree correlations (model 2).

The dependence of $q_{c}$ on $\alpha$ for the uncorrelated network provides a reference for the understanding of the behaviors of models 1 and 2 . In both models the percolation threshold increases for increasing localization and saturates for small $\alpha$. A contrasting picture for the two models appears in the dependence on assortativity. For model 1 networks, the percolation threshold decreases with increasing $r$, while it increases for model 2 networks. Indeed, assortative model 1 networks percolate more easily because high-degree nodes tend to connect to a subnetwork which spans the whole spatial domain and is resilient to random removal of links. Thus, the effect on percolation in this case is comparable to assortativity in nonspatial random networks, where the percolation threshold is found to decrease or even vanish [35-42].

For model 2, the prior segregation of nodes by degree results in an interesting effect. With weak spatial correlations $(\alpha \simeq 0$ ), model 2 is very similar to the uncorrelated network, possibly because long-range connections dominate degree correlations. For $\alpha \simeq-3$, spatial constraints favor short-range connections while still maintaining long-range connectivity. This architecture facilitates the interconnectivity of the high degree nodes, which are spatially close after segregation, giving rise to a subnetwork of highly connected nodes that extends the entire system, slightly reducing the percolation threshold. In contrast, for $\alpha \simeq-10$, short-range connections become dominant, giving rise to weakly interconnected modules of high degree nodes, a configuration that substantially increases the percolation threshold. Note that spatial segregation, which places high-degree nodes close to one another, is crucial in shaping such a network. Nodes indeed become inhomogeneously distributed in space and form dense connectivity clusters that are separated by depleted areas (see Fig. 6). These subnetworks easily disconnect from one another when links are randomly removed, leading to the observed higher percolation threshold.

In the comparison between the two models we have excluded the disassortative case of model 1 since model 2 cannot generate disassortative topologies. In general, however, we have observed that disassortative networks do not significantly differ in percolation behavior from spatially embedded ER networks.

\section{CONCLUSION}

The spatial embedding of complex networks is often found to play a role that is more important than initially thought. Prominent examples are power grids, transportation, and mobile phone networks [25]. Here the knowledge of topology 
is not sufficient and the extraction of network properties can be misleading when metric properties are ignored. In neuronal tissues, the importance of spatial embedding has been often ignored in the analysis of their formation, structure, and function. Indeed, the study of spatially embedded neuronal systems may not only provide a good example for the combination of metric properties with graph theory, but also advance our understanding of spatial networks in general. An attractive system is a neuronal network in culture, which is typically grown on a two-dimensional substrate [12] or patterned to follow specific circuits and designs [52]. The topological features of standard, two-dimensional cultures were studied experimentally and theoretically within a percolation framework [13-15], although in the context of nonspatial graphs. These studies concluded that the neurons' degree distribution was Poisson-like, which supports an Erdős-Rényi description of the neuronal network. However, it may be possible that the connectivity degree distribution and the distribution of connectivity lengths substantially change with the spatial location of the neurons and the wiring of connections, as suggested from experiments in patterned neuronal networks [53-56].

Our models presented here take into account more complex features of the network that may provide a better description of topological features in such cultures. This regards not only the spatial embedding of the network, but also the possibility that the network exhibits correlations in the degree of the nodes, which have been found in some neuronal networks $[57,58]$. We tackle the issue by numerical percolation analysis for random networks with power law length distributions and degree correlations.

Our results are twofold. First, the impact of spatial constraints on degree-correlated random networks is qualitatively similar to the uncorrelated case. As Li et al. [44] recently showed, spatial constraints generally increase the percolation threshold and shift the percolation transition from the universality class of random graphs to the universality class of two-dimensional lattices. Second, assortativity decreases the percolation threshold of spatial random graphs when nodes are distributed randomly in space, whereas assortativity increases percolation when nodes are spatially segregated by their degree. Therefore, regarding percolation, degree correlations cannot be treated as an independent topological feature, but must be taken into account together with the geographical properties of the network.

Our models may be valuable in other contexts, including the resilience of the network to insult and the interplay between topology and dynamics. Hence, in the future, and following the experimental concept in Breskin et al. [13], we aim at carrying out experiments in neuronal cultures with distinct architectures to test and refine our study. We foresee that the comparison of the experimental and numerical curves of the giant component $G(q, N)$ will provide a valuable insight on the existence of assortativity an degree correlation in neuronal cultures. Additionally, recent studies have provided tools for the reconstruction of connectivity in neuronal cultures from activity data [59], which allows the identification of degree correlations and the characteristics of connectivity length distributions. This information will be highly valuable to test the validity of our models and their applicability to experimental data. Our ultimate goal is to comprehend the interplay between connectivity degree correlations, percolation threshold, and neuronal network dynamics.

Finally, we would like to stress that our findings could be of importance for further systems. For example, in disease spreading, research focuses on the resilience of social networks. Social networks usually show assortative degree correlations $[35,36]$ and evolve under geographical constraints [25]. The idea that social networks are resilient to failure because they are assortative could be reexamined, taking into account their geographical characteristics.

\section{ACKNOWLEDGMENTS}

C.S., I.S., and S.R. were supported by the Deutsche Forschungsgemeinschaft (IRTG 1740). J.S. acknowledges the financial support of MCI (Spain) under Projects No. FIS200907523 and No. FIS2010-21924-C02-02, and the Generalitat de Catalunya (Spain) under Project No. 2009-SGR-14.
[1] S. R. Broadbent and J. M. Hammersley, in Mathematical Proceedings of the Cambridge Philosophical Society (Cambridge University Press, Cambridge, England, 1957), Vol. 53, pp. 629-641.

[2] S. N. Dorogovtsev, A. V. Goltsev, and J. F. Mendes, Rev. Mod. Phys. 80, 1275 (2008).

[3] B. I. Shklovskii, H. Fritzsche, and S. D. Baranovskii, Phys. Rev. Lett. 62, 2989 (1989).

[4] M. Bär, M. Falcke, H. Levine, and L. S. Tsimring, Phys. Rev. Lett. 84, 5664 (2000).

[5] E. Agliari, A. Annibale, A. Barra, A. C. C. Coolen, and D. Tantari, J. Phys. A: Math. Theor. 46, 335101 (2013).

[6] E. Agliari and A. Barra, Europhys. Lett. 94, 10002 (2011).

[7] L. Sander, C. Warren, I. Sokolov, C. Simon, and J. Koopman, Math. Biosci. 180, 293 (2002).
[8] S. Solomon, G. Weisbuch, L. de Arcangelis, N. Jan, and D. Stauffer, Phys. A (Amsterdam, Neth.) 277, 239 (2000).

[9] E. Agliari, C. Cioli, and E. Guadagnini, Phys. Rev. E 84, 031120 (2011).

[10] J. Nagler, A. Levina, and M. Timme, Nat. Phys. 7, 265 (2011).

[11] D. Achlioptas, R. M. D'Souza, and J. Spencer, Science 323, 1453 (2009).

[12] J.-P. Eckmann, O. Feinerman, L. Gruendlinger, E. Moses, J. Soriano, and T. Tlusty, Phys. Rep. 449, 54 (2007).

[13] I. Breskin, J. Soriano, E. Moses, and T. Tlusty, Phys. Rev. Lett. 97, 188102 (2006).

[14] J. Soriano, M. Rodríguez Martínez, T. Tlusty, and E. Moses, Proc. Natl. Acad. Sci. 105, 13758 (2008).

[15] O. Cohen, A. Keselman, E. Moses, M. R. Martínez, J. Soriano, and T. Tlusty, Europhys. Lett. 89, 18008 (2010).

[16] H. Amini, J. Stat. Phys. 141, 459 (2010). 
[17] T. Tlusty and J.-P. Eckmann, J. Phys. A: Math. Theor. 42, 205004 (2009).

[18] S. Jacobi, J. Soriano, M. Segal, and E. Moses, European Journal of Neuroscience 30, 998 (2009).

[19] R. Albert and A.-L. Barabási, Rev. Mod. Phys. 74, 47 (2002).

[20] L. Sander, C. Warren, and I. Sokolov, Phys. A (Amsterdam, Neth.) 325, 1 (2003).

[21] M. Barthélemy, Europhys. Lett. 63, 915 (2003).

[22] S. S. Manna and P. Sen, Phys. Rev. E 66, 066114 (2002).

[23] R. Xulvi-Brunet and I. M. Sokolov, Phys. Rev. E 66, 026118 (2002).

[24] C. P. Warren, L. M. Sander, and I. M. Sokolov, Phys. Rev. E 66, 056105 (2002).

[25] M. Barthélemy, Phys. Rep. 499, 1 (2011).

[26] Y. Hayashi, IPSJ Digital Courier 2, 155 (2006).

[27] P. E. Vértes, A. F. Alexander-Bloch, N. Gogtay, J. N. Giedd, J. L. Rapoport, and E. T. Bullmore, Proc. Natl. Acad. Sci. 109, 5868 (2012).

[28] J. G. Orlandi, J. Soriano, E. Alvarez-Lacalle, S. Teller, and J. Casademunt, Nat. Phys. 9, 582 (2013).

[29] R. Lambiotte, V. Blondel, C. De Kerchove, E. Huens, C. Prieur, Z. Smoreda, and P. Van Dooren, Phys. A (Amsterdam, Neth.) 387, 5317 (2008).

[30] D. Brockmann, L. Hufnagel, and T. Geisel, Nature (London) 439, 462 (2006).

[31] D. Liben-Nowell, J. Novak, R. Kumar, P. Raghavan, and A. Tomkins, Proc. Natl. Acad. Sci. USA 102, 11623 (2005).

[32] S. Yook, H. Jeong, and A. Barabási, Proc. Natl. Acad. Sci. 99, 13382 (2002).

[33] G. Bianconi, P. Pin, and M. Marsili, Proc. Natl. Acad. Sci. 106, 11433 (2009).

[34] A. Barrat, M. Barthélemy, and A. Vespignani, J. Stat. Mech.: Theory Expt. (2005) P05003.

[35] M. E. J. Newman, Phys. Rev. Lett. 89, 208701 (2002).

[36] M. E. J. Newman, Phys. Rev. E 67, 026126 (2003).

[37] R. Xulvi-Brunet and I. M. Sokolov, Phys. Rev. E 70, 066102 (2004).

[38] R. Xulvi-Brunet, Ph.D. thesis, Humboldt Universität zu Berlin, Berlin, 2007.
[39] M. Boguñá, R. Pastor-Satorras, and A. Vespignani, Phys. Rev. Lett. 90, 028701 (2003).

[40] A. Vázquez and Y. Moreno, Phys. Rev. E 67, 015101 (2003).

[41] J. D. Noh, Phys. Rev. E 76, 026116 (2007).

[42] A. V. Goltsev, S. N. Dorogovtsev, and J. F. F. Mendes, Phys. Rev. E 78, 051105 (2008).

[43] M. Rubinov and O. Sporns, Neuroimage 52, 1059 (2010).

[44] D. Li, G. Li, K. Kosmidis, H. Stanley, A. Bunde, and S. Havlin, Europhys. Lett. 93, 68004 (2011).

[45] M. E. J. Newman, S. H. Strogatz, and D. J. Watts, Phys. Rev. E 64, 026118 (2001).

[46] P. Erdős and A. Rényi, Publicationes Mathematicae Debrecen 6, 290 (1959).

[47] J. Badham and R. Stocker, Journal of Artificial Societies and Social Simulation 13, 11 (2010).

[48] D. Stauffer and A. Aharony, Introduction To Percolation Theory (Taylor \& Francis, London, 1994).

[49] A. Bunde and S. Havlin, Fractals and Disordered Systems (Springer, New York, 1991).

[50] L. Daqing, K. Kosmidis, A. Bunde, and S. Havlin, Nat. Phys. 7, 481 (2011).

[51] H. Hong, M. Ha, and H. Park, Phys. Rev. Lett. 98, 258701 (2007).

[52] M. Shein-Idelson, E. Ben-Jacob, and Y. Hanein, Frontiers in Neuroengineering 4, 10 (2011).

[53] C. Wyart, C. Ybert, L. Bourdieu, C. Herr, C. Prinz, and D. Chatenay, J. Neurosci. Methods 117, 123 (2002).

[54] T. Gabay, E. Jakobs, E. Ben-Jacob, and Y. Hanein, Phys. A (Amsterdam, Neth.) 350, 611 (2005).

[55] S. Teller and J. Soriano, in AIP Conference Proceedings (American Institute of Physics, College Park, MD, 2013), Vol. 1510, p. 244.

[56] E. Tibau, C. Bendiksen, S. Teller, N. Amigó, and J. Soriano, in AIP Conference Proceedings (American Institute of Physics, College Park, MD, 2013), Vol. 1510, p. 54.

[57] L. M. A. Bettencourt, G. J. Stephens, M. I. Ham, and G. W. Gross, Phys. Rev. E 75, 021915 (2007).

[58] D. J. Watts and S. H. Strogatz, Nature (London) 393, 440 (1998).

[59] O. Stetter, D. Battaglia, J. Soriano, and T. Geisel, PLoS Computational Biology 8, e1002653 (2012). 